\title{
DESENVOLVIMENTO E AVALIAÇÃO DE UM ANEMÔMETRO DE FIO QUENTE OPERANDO À TEMPERATURA CONSTANTE ${ }^{1}$
}

\author{
Carlos Augusto de P. Sampaio², Evandro Ferreira Passos ${ }^{3}$, \\ Gutemberg Pereira Dias ${ }^{4}$ e Paulo César Correa ${ }^{4}$
}

\begin{abstract}
RESUMO
Esta pesquisa teve como objetivo desenvolver um anemômetro de fio quente para operar à temperatura constante usando-se, como elemento sensível, filamento de tungstênio de lâmpada incandescente, como braço da ponte de Wheatstone. Um circuito eletrônico foi construído e testado, permitindo controlar a tensão no sensor e restaurar o equilíbrio da ponte de Wheatstone em processos de troca de calor pelo sensor. Os resultados mostraram que a corrente elétrica e a temperatura de operação do sensor, mediante compromisso entre vida útil e sensibilidade, foram de $160 \mathrm{~mA} \mathrm{e} 140^{\circ} \mathrm{C}$, respectivamente; a calibração com o sensor no circuito ponte de Wheatstone mostrou sensibilidade para medir a velocidade do ar no intervalo de 0,00 e $5,00 \mathrm{~m} \cdot \mathrm{s}^{-1}$ e que o circuito eletrônico apresentou saturação para velocidades do ar inferiores a $0,50 \mathrm{~m} \cdot \mathrm{s}^{-1}$.
\end{abstract}

Palavras-chave: anemômetro de temperatura constante, sensor de tungstênio, ponte de Wheatstone

\section{DEVELOPMENT AND EVALUATION OF A HOT-WIRE ANEMOMETER OPERATING AT CONSTANT TEMPERATURE}

\begin{abstract}
This research was carried out with the purpose of developing a hot-wire anemometer operating at constant temperature, using as a sensitive element a tungsten filament fan incandescent lamp as a Wheatstone bridge arm. An eletronic circuit was constructed and tested to control the voltage in the sensor and to restore the equilibrium of the Wheatstone bridge in the transfer of heat by the sensor. The results showed that the sensor's eletrical current and temperature of operation, considering useful life and sensibility, was $160 \mathrm{~mA}$ and $140^{\circ} \mathrm{C}$, respectively. The calibration with the sensor in the Wheatstone bridge circuit showed sensibility to measure the air velocity in the interval between 0.00 and $5.00 \mathrm{~m} . \mathrm{s}^{-1}$. It also showed that this eletronic circuit presented saturation in measuring the air velocity lower than $0.50 \mathrm{~m} \cdot \mathrm{s}^{-1}$.
\end{abstract}

Key words: constant temperature anemometer, tungsten sensor, Wheatstone bridge

\section{INTRODUÇÃO}

Anemômetros são instrumentos usados para medidas de velocidade de ar e de outros fluidos; existem vários com características de construção e operação diferentes. Para baixas velocidades, os anemômetros que operam no princípio das trocas de calor são os mais indicados e seu funcionamento se baseia nas trocas de calor entre sensor aquecido e o fluido, provocando alteração da resistência elétrica do sensor, que pode ser correlacionada à velocidade do fluido. Estes anemômetros

\footnotetext{
${ }^{1}$ Parte da tese de mestrado do primeiro autor. Fone (031)899-2734, Viçosa, MG

2 Professor M.Sc, da Faculdade de Agronomia do Centro de Ciência Veterinaria - CAV, Universidade do Estado de Santa Catarina - UDESC, CP 381, CEP 88.520-000, Lages, SC, fone (049)225-2866, fax (049)225-3401. E-mail a2caps@ cav.udesc.br

3 Prof. Titular, Departamento de Física, UFV, Viçosa, MG, fone (031) 899- 2799

${ }^{4}$ Prof. Adjunto, Departamento de Eng. Agrícola, UFV, Viçosa, MG, fone (031) 899- 2743
} 
possuem alta sensibilidade, baixa velocidade de partida e rápido tempo de resposta, podendo ser de fio ou filme quente, conforme relatam Platt \& Griffiths (1972); Rosenberg et al. (1983); Miller et al. (1987); Pearcy et al. (1989).

O fenômeno físico mais importante que ocorre entre o sensor aquecido e o fluido é a troca de calor. Para este estudo, considera-se o filamento como cilindro infinitesimal; assim, o balanço de energia relativo às trocas de calor entre o sensor e o fluido para regime transiente e fluxo unidirecional, é mostrado na Figura 1.

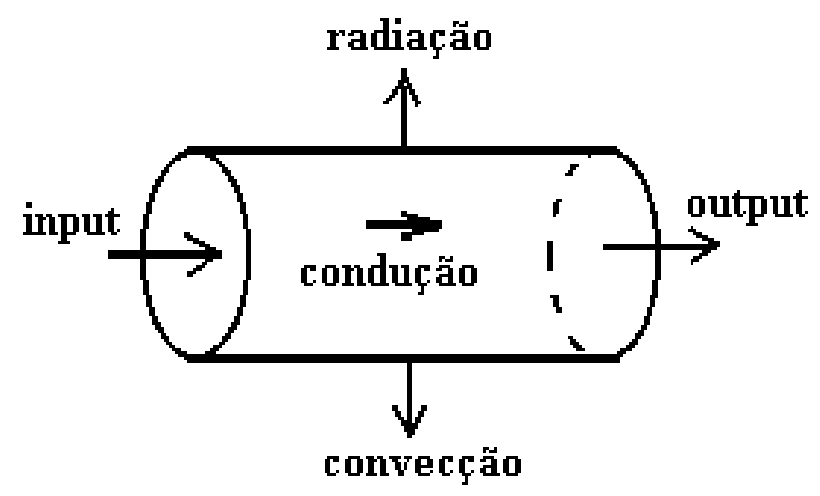

Figura 1. Elemento infinitesimal do sensor e as trocas de calor existentes

\section{Condução}

energia input no sensor

$$
\mathrm{qc}_{1}=\mathrm{Ks} .\left.\mathrm{A} \cdot \frac{\delta \mathrm{Ts}}{\delta \mathrm{x}}\right|_{\mathrm{x}}
$$

energia output no sensor

$$
\mathrm{q}_{\mathrm{c} 2}=\left.\mathrm{K} \cdot \mathrm{A} \cdot \frac{\delta \mathrm{T}_{\mathrm{s}}}{\delta_{\mathrm{x}}}\right|_{\mathrm{x}+\mathrm{dx}}
$$

O balanço de troca de calor por condução resulta em:

$$
\mathrm{q}=-\mathrm{K}_{\mathrm{s}} \mathrm{A} \frac{\delta \mathrm{T}_{\mathrm{s}}}{\delta \mathrm{s}}-\mathrm{A} \frac{\delta}{\delta}\left(\mathrm{K}_{\mathrm{s} \cdot} \frac{\delta T_{\mathrm{s}}}{\delta \mathrm{k}}\right) \mathrm{dx}
$$

em que:

$\mathrm{K}_{\mathrm{s}}$ - Coeficiente de condutibilidade térmica do sensor, W. $\mathrm{m}^{-1} \cdot \mathrm{K}^{-1}$ A - Área da seção transversal do sensor, $\mathrm{m}^{2}$

$\mathrm{T}_{\mathrm{s}}$ - Temperatura do sensor, $\mathrm{K}$.

\section{Convecção}

$$
\mathrm{q}_{\mathrm{cv}}=\pi \cdot d \cdot h \cdot\left(\mathrm{T}_{\mathrm{s}}-\mathrm{T}_{\mathrm{f}}\right) \mathrm{d}_{\mathrm{x}}
$$

em que:

h - Coeficiente de troca calor por convecção, W.m. ${ }^{-2} \cdot K^{-1}$

$\mathrm{T}_{\mathrm{f}}$ - Temperatura do fluido, $\mathrm{K}$.

d - Diâmetro do sensor, $m$

\section{Radiação}

em que:

$$
\mathrm{q}_{\mathrm{r}}=\pi \cdot \mathrm{d} \cdot \sigma \cdot \varepsilon \cdot\left(\mathrm{T}_{\mathrm{s}}^{4}-\mathrm{T}_{\mathrm{v}}^{4}\right) \mathrm{d}_{\mathrm{x}}
$$

$\sigma$ - Constante de Stefan-Boltzmann, 5,67.10-8 W. $\mathrm{m}^{-2} \cdot \mathrm{K}^{-4}$

$\varepsilon$ - Emissividade da superfície do sensor, adimensional

$\mathrm{T}_{\mathrm{v}}$ - Temperatura da vizinhança do sensor, $\mathrm{K}$.

\section{Aquecimento do sensor}

$$
q_{g}=\frac{I^{2} \rho_{s}}{A} d x
$$

em que

I - Corrente elétrica de aquecimento do sensor, A

$\rho_{\mathrm{s}}$ - Resistividade do sensor, W.m. $\mathrm{A}^{-2}$.

\section{Variação de energia interna no sensor}

$$
\mathrm{E}=\rho . \mathrm{c} \cdot \mathrm{A} \cdot \frac{\delta \mathrm{T}_{\mathrm{s}}}{\delta \mathrm{t}} \mathrm{d}_{\mathrm{x}}
$$

em que

$\rho$ - Massa específica do sensor, $\mathrm{kg} \cdot \mathrm{m}^{-3}$

c - Calor específico do sensor, J.kg-1 $\mathrm{K}^{-1}$

t - Tempo, s.

$\mathrm{O}$ balanço de energia no sensor resulta em:

$\mathrm{A} \frac{\delta}{\delta \mathrm{x}}\left(\mathrm{K}_{\mathrm{s}} \frac{\delta \mathrm{T}_{\mathrm{s}}}{\delta \mathrm{x}}\right)+\frac{\mathrm{I}^{2} \rho_{\mathrm{s}}}{\mathrm{A}}-\rho . \mathrm{c} \cdot \mathrm{A} \frac{\delta \mathrm{T}_{\mathrm{s}}}{\delta \mathrm{t}}-\pi \cdot \mathrm{d}\left[\mathrm{h}\left(\mathrm{T}_{\mathrm{s}}-\mathrm{T}_{\mathrm{f}}\right)+\delta \cdot \varepsilon\left(\mathrm{T}_{\mathrm{s}}^{4}-\mathrm{T}_{\mathrm{v}}^{4}\right)\right]=0$

Segundo Champagne et al. (1967); Ower \& Pankhurst, (1977) e Lomas, (1986) pode-se considerar o regime de troca de calor como permanente, devido à pequena massa do sensor $\mathrm{e}$ predominância da convecção. Assim, a Equação 8 será escrita:

$$
\frac{I^{2} \rho_{S}}{A}=\pi \cdot d \cdot h\left(T_{S}-T_{f}\right)
$$

De acordo com Champagne et al. (1967) a distribuição da temperatura no sensor aquecido no interior de um escoamento de fluido é simétrica, ocorrendo a máxima temperatura no plano médio para qualquer relação entre comprimento e diâmetro do sensor.

Ower \& Pankhurst (1977) e Lomas (1986) constataram que a convecção natural do ar torna-se significativa para baixas velocidades, dificultando a medida do escoamento. O limite de escoamento em que a convecção natural se torna significativa é dada pela expressão:

$$
\operatorname{Re}<\left(g \cdot T_{s} . d^{3}\right)^{1 / 3} \cdot\left(T_{f} . v^{2}\right)^{-1 / 3}
$$

em que

Re - Número de Reynolds

g - Aceleração da gravidade, $\mathrm{m} \cdot \mathrm{s}^{-2}$

d - Diâmetro do sensor, $m$

V - Viscosidade cinemática do fluido, $\mathrm{m}^{2} \cdot \mathrm{s}^{-1}$

De acordo com Lomas (1986) a máxima potência dissipada pelo sensor dá-se com incidência normal do fluido (Figura 2).

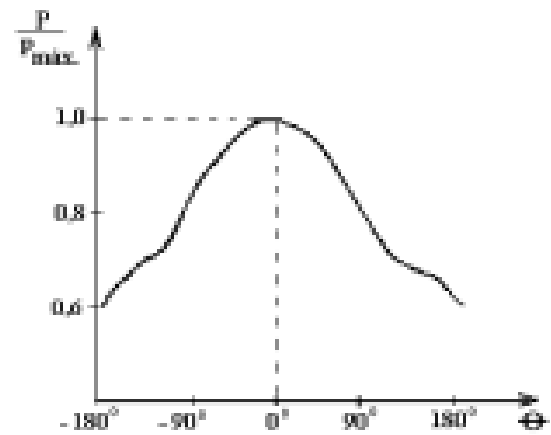

Figura 2. Potência dissipada pelo sensor para diferentes incidências do fluido 
Na Figura 2, o eixo vertical representa a razão entre a potência momentânea e a potência máxima dissipada, enquanto a inclinação do sensor no escoamento é representada na abscissa.

A maioria dos sensores de medida de velocidade do ar é metálica, sendo os mais utilizados os de tungstênio, platina e suas ligas. Os metais possuem coeficiente de resistência elétrica positivo com a temperatura e esta relação é escrita pela equação:

$$
\left.\mathrm{R}_{\mathrm{s}}=\mathrm{R}_{\mathrm{o}}\left[1+\alpha_{\mathrm{o}}\left(\mathrm{T}_{\mathrm{s}}-\mathrm{T}_{\mathrm{o}}\right)+\alpha_{1}\left(\mathrm{~T}_{\mathrm{s}}-\mathrm{T}_{\mathrm{o}}\right)^{2}+\ldots\right)\right]
$$

em que

$\mathrm{R}_{\mathrm{s}}$ - Resistência do sensor aquecido, W

$\mathrm{T}_{\mathrm{s}}$-Temperatura do sensor aquecido, ${ }^{\circ} \mathrm{C}$

$\mathrm{R}_{\mathrm{o}}$ - Resistência do sensor à temperatura de referência, W

$\mathrm{T}_{\mathrm{o}}$ - Temperatura de referência, geralmente $0^{\circ} \mathrm{C}$.

O tungstênio possui $\alpha_{0}$ de $5,20.10^{-3}{ }^{\circ} \mathrm{C}^{-1}$ e $\alpha_{1}$ de $7,00 \times 10^{-7}{ }^{\circ} \mathrm{C}^{-1}$, é empregado como sensor, devido às propriedades de resistência mecânica e temperatura de fusão alta. Como desvantagem, existe a facilidade de oxidação pelo ar, limitando seu aquecimento a temperatura de $300^{\circ} \mathrm{C}$.

A Ponte de Wheatstone é um circuito composto de resistores e é usada para determinar a resistência elétrica dos componentes elétricos e detectar pequenas variações nas resistências desses componentes (Figura 3).

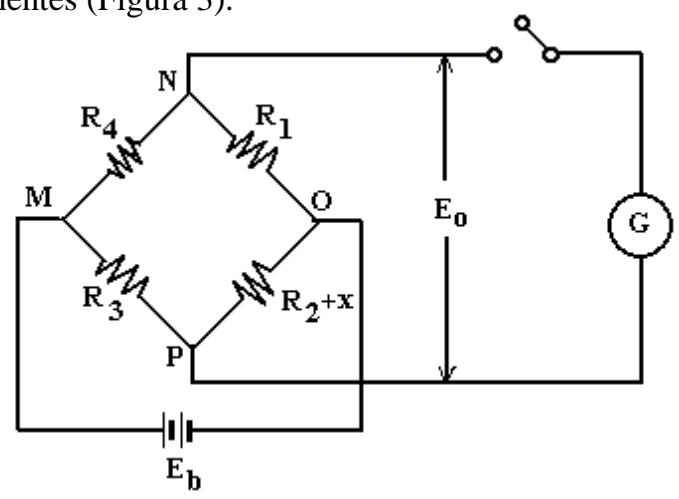

Figura 3. Ponte de Wheatstone

A ponte está equilibrada eletricamente quando os potenciais elétricos em N e P se igualam, considerando-se isentas influências elétricas externas à ponte.

A tensão elétrica $\mathrm{E}_{\mathrm{o}}$ entre os terminais $\mathrm{N}$ e $\mathrm{P}$ em uma ponte não equilibrada eletricamente é escrita da seguinte forma:

$$
E_{o}=E_{b}\left[R_{4}\left(R_{1}+R_{4}\right)^{-1}-R_{3}\left(\left(R_{2}+x\right)+R_{3}\right)^{-1}\right]
$$

em que

$\mathrm{E}_{\mathrm{b}}$ - Fonte de tensão elétrica

$\mathrm{R}_{1}, \mathrm{R}_{2}, \mathrm{R}_{3}, \mathrm{R}_{4}$ - Resistores

$\mathrm{X}$ - Representa a variação de resistência do resistor $\mathrm{R}_{2}$.

O cálculo de desequilíbrio da ponte é analisado pelo circuito de Thevenin (Figura 4).
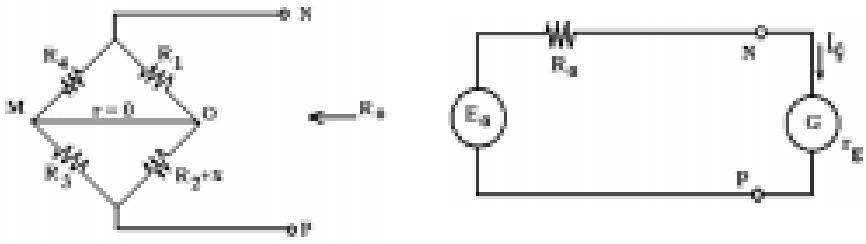

Figura 4. Resistência interna do circuito de Thevenin e circuito equivalente
Pelo teorema de Thevenin, a resistência interna equivalente $\mathrm{R}_{\mathrm{o}}$ e a corrente no galvanômetro $(\mathrm{G})$ para o desequilíbrio serão:

$$
\begin{gathered}
\mathrm{R}_{\mathrm{o}}=\left\{\left[\left(\mathrm{R}_{4} \cdot \mathrm{R}_{1}\right) \cdot\left(\mathrm{R}_{4}+\mathrm{R}_{1}\right)^{-1}\right]+\left[\mathrm{R}_{3} \cdot\left(\mathrm{R}_{2}+\mathrm{x}\right) \cdot\left(\mathrm{R}_{3}+\mathrm{R}_{2}+\mathrm{x}\right)^{-1}\right]\right\} \\
\mathrm{I}_{\mathrm{g}}=\mathrm{E}_{\mathrm{o}} \cdot\left(\mathrm{R}_{\mathrm{o}}+\mathrm{r}_{\mathrm{g}}\right)^{-1}
\end{gathered}
$$

De acordo com Davis (1970); Perry \& Morrison (1971); Weidman \& Browand (1975); Ower \& Pankhurst (1977); Lomas (1986); Miller et al (1987); Pearcy et al. (1989); Watmuff (1995) o anemômetro de fio quente de temperatura constante caracterizase pelo aquecimento do sensor por determinada corrente elétrica, seu resfriamento devido às trocas de calor com o fluido e mantença da resistência e temperatura média do sensor a valores praticamente constantes, por um sistema de realimentação, que fornece a corrente elétrica de aquecimento, variável com a velocidade do fluido (Figura 5).

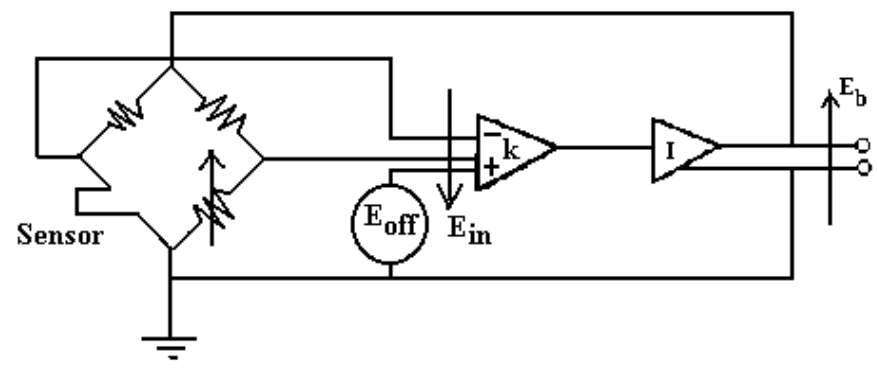

Figura 5. Diagrama do circuito de temperatura constante

A compensação, devido às mudanças de temperatura do fluido, é realizada por vários métodos, como ajustes gráficos, equações, fator de correção e circuito eletrônico de compensação. O uso de compensador de temperatura como braço da ponte, com as mesmas características do sensor e submetido às mesmas variações de temperatura, é o método mais indicado; assim, os efeitos térmicos se produzem nos dois lados da ponte e se compensarão.

Conforme Rosenberg (1983) Lomas (1986); Pearcy et al. (1989); a maioria dos sensores é calibrada em túnel de vento, com uso do tubo de Pitot e micromanômetro.

Os objetivos deste trabalho foram:

a) avaliar as propriedades elétricas de filamentos de tungstênio de pequenas lâmpadas incandescentes, como sensor de anemômetro de fio quente;

b) verificar a resposta do sensor ligado em ponte de Wheatstone, para indicação da velocidade do ar;

c) obter a construção e resposta de um circuito eletrônico de realimentação da ponte de Wheatstone.

\section{MATERIAL E MÉTODOS}

O presente trabalho experimental foi realizado no Laboratório de Instrumentação do Departamento de Física, da Universidade Federal de Viçosa.

Os ensaios foram divididos em duas etapas: na primeira, procedeu-se à caracterização elétrica e escolha de filamentos de lâmpada incandescente, para serem utilizados como sensor do anemômetro; na segunda, verificou-se a resposta do sensor ligado em ponte de Wheatstone, com circuito eletrônico de realimentação da ponte. 
Para a realização dos ensaios, construíram-se dois equipamentos: túnel de vento e cilindro com ar ascendente, mostrados nas Figuras 6 e 7, respectivamente. O túnel de vento foi construído de acordo com as normas NAFM (1952).

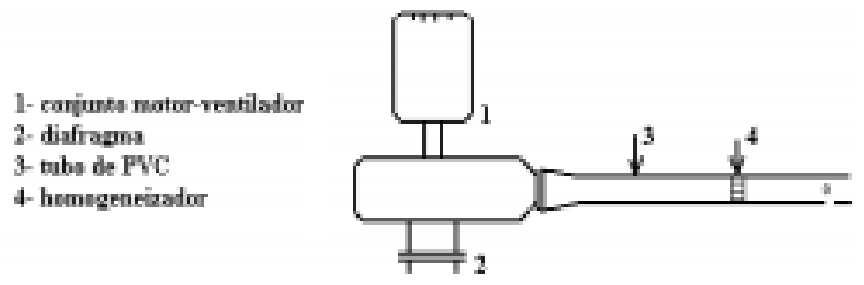

Figura 6. Túnel de vento usado nos ensaios de calibração do anemômetro

1- conjunto motor-ventilador

2- tubo de PVC

3- homogeneizador

4- resistência elétrica

5- saída de ar

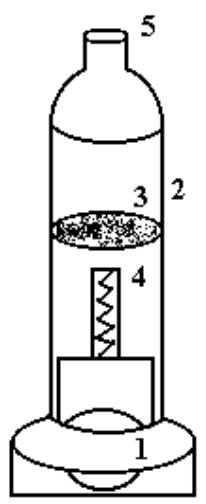

Figura 7. Cilindro de ar ascendente para caracterização do filamento

As velocidades instantâneas do ar foram medidas através de um anemômetro de fio quente (padrão), E. Schiltknecht Ing. Sia., modelo Thermo-Air I e por tubo de Pitot, CAT 160, no 8, com micromanômetro de $0,20 \mathrm{~mm}$ ca entre escalas. Na aferição do anemômetro padrão com o de tubo de Pitot para velocidades de ar no intervalo de 0,0 a 5,00m. $\mathrm{s}^{-1}$, obteve-se o coeficiente de regressão, $\mathrm{R}^{2}$ de 0,99 .

As grandezas elétricas envolvidas: resistência, tensão e corrente elétrica e a temperatura do ar, foram obtidas por um sistema de aquisição de dados Hewlett Packard, modelo 3421A. A corrente elétrica de aquecimento do sensor foi determinada medindo-se a queda de tensão nos extremos de um fio de constantan de resistência elétrica conhecida, em série com o circuito do sensor, e a temperatura do ar medida com termopar cobre-constantan.

\section{Sensor}

O sensor foi definido pelas características de sensibilidade, resistência mecânica e ponto de fusão, escolhendo-se o filamento de tungstênio de lâmpada incandescente com características elétricas de $2,5 \mathrm{~V}$ e $0,3 \mathrm{~A}$, com $2,50 \mathrm{~mm}$ de comprimento e $0,08 \mathrm{~mm}$ de diâmetro, resistência elétrica a frio e em operação de $8,33 \Omega$ e $1,25 \Omega$, respectivamente.

A compensação devido às variações de temperatura do ar foi conseguida por um sensor passivo, com as mesmas características elétricas do sensor ativo e submetido às mesmas variações de temperatura.

A temperatura de operação do sensor foi obtida colocando-se o sensor em banho de óleo, que é aquecido, monitorando-se a temperatura do óleo e a variação da resistência do sensor, simultaneamente; esta temperatura, em que a resistência do sensor se igualou à sua resistência de operação obtida pela lei de Ohm, corresponde à temperatura de operação do sensor.
$\mathrm{O}$ aquecimento do sensor foi obtido submetendo-o a correntes de 100, 150, 160, 170, 180 e 200mA, limitada pela sensibilidade e pela vida útil e em fluxos de ar de 0,0 a 5,00m. $\mathrm{s}^{-1}$. A vida útil foi determinada verificando-se a variação da resistência elétrica do sensor com o tempo para a corrente elétrica de aquecimento preestabelecida.

O efeito da temperatura do ar entre $19 \mathrm{a} 41^{\circ} \mathrm{C}$ sobre o sensor foi medido para uma velocidade constante de $2,40 \mathrm{~m} \cdot \mathrm{s}^{-1}$.

\section{Resposta ao cosseno}

A resposta ao cosseno foi verificada variando-se o ângulo formado entre o sensor e o escoamento. A incidência do escoamento normal ao sensor foi considerada como sendo de $90^{\circ}$.

\section{Circuito eletrônico}

$\mathrm{O}$ anemômetro de temperatura constante usa circuito em ponte de Wheatstone com amplificador operacional de realimentação da ponte ( $\mathrm{Lm} 324)$. A ponte de Wheatstone é formada pelo sensor, por três resistores de constantan, por um potenciômetro e um resistor compensador de temperatura, enquanto o circuito eletrônico é composto por um circuito integrado associado a componentes ativos e passivos, que regem o ganho e a polarização do operacional e do transistor (Figura 8).

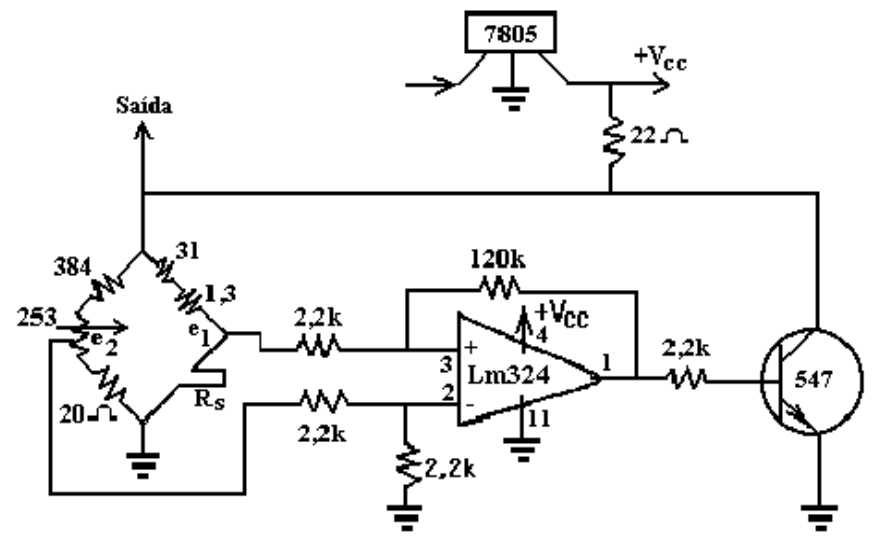

Figura 8. Ponte de Wheatstone e circuito de temperatura constante

A voltagem na ponte, denotada por $\mathrm{e}_{1}$ e e $\mathrm{e}_{2}$, é fornecida por uma fonte não simétrica com retificação de onda completa, tensão de saída de 9,0V, ligada a um regulador de tensão elétrica. A ponte de Wheatstone é conectada ao amplificador operacional em circuito denominado amplificador-inversor, fechado com realimentação negativa de tensão, ligado a um transistor de baixa potência em configuração emissor comum, que realimenta a ponte, restaurando seu equilíbrio. O sinal emitido pelo operacional para restaurar o equilíbrio da ponte é proporcional à variação da resistência do sensor, que está correlacionado à velocidade do fluido.

Foram determinadas as curvas fornecidas pelo protótipo para velocidades do ar entre 0,0 a $5,00 \mathrm{~m} . \mathrm{s}^{-1}$, com o sensor alinhado perpendicularmente ao fluxo.

\section{RESULTADOS E DISCUSSÃO}

\section{Aquecimento, temperatura de operação e vida útil do sensor}

Observou-se que, para a corrente de $100 \mathrm{~mA}$, houve baixa sensibilidade do sensor e, para $200 \mathrm{~mA}$, constatou-se a sua queima. Para as correntes de 150,160, 170 e 180mA, as respostas quanto à sensibilidade foram semelhantes escolhendo-se, portanto, a corrente de $160 \mathrm{~mA}$, mediante características de sensibilidade e vida útil do sensor. 
A temperatura do sensor na potência de dissipação máxima foi de $140^{\circ} \mathrm{C}$ e pela Equação 11 foi de $110^{\circ} \mathrm{C}$, diferença esta que pode ser devida à presença de impurezas na composição do filamento.

Os testes de vida útil mostraram que, após 153,8 horas de uso, a resistência elétrica do sensor permaneceu praticamente constante.

\section{Resposta ao cosseno}

A dissipação de calor pelo sensor para diferentes ângulos de incidência do fluido para velocidades do ar de 0,70 e $3,90 \mathrm{~m} \cdot \mathrm{s}^{-1}$ está mostrada na Figura 9. Verificou-se baixa sensibilidade para velocidades inferiores a $0,70 \mathrm{~m} \cdot \mathrm{s}^{-1} \mathrm{e}$ acima de $3,90 \mathrm{~m} \cdot \mathrm{s}^{-1}$, enquanto a máxima sensibilidade ocorreu entre 0,70 a $3,90 \mathrm{~m} \cdot \mathrm{s}^{-1}$. Verificouse, também, maior potência dissipada pelo sensor com incidência normal do fluido e, em incidências paralelas, a potência dissipada é mínima.
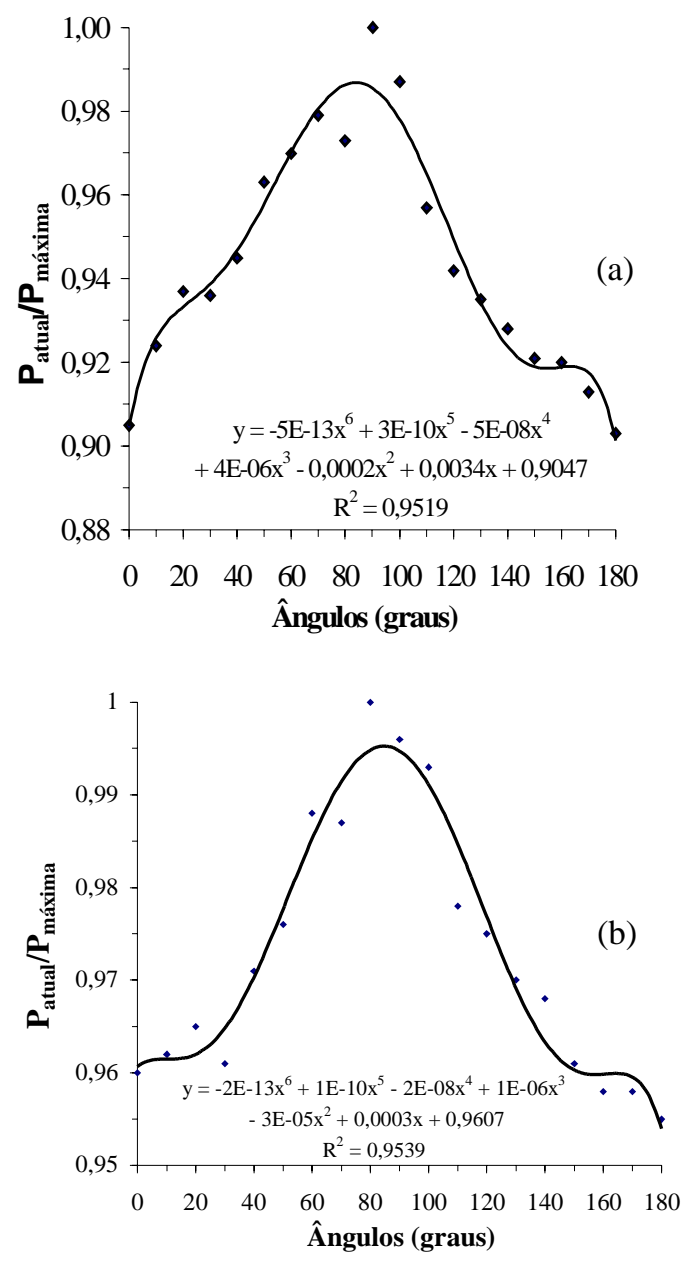

Figura 9. Potência dissipada pelo sensor para diferentes incidências do fluido e para velocidades do ar de $0,70 \mathrm{~m} \cdot \mathrm{s}^{-1}$ (a) e $3,90 \mathrm{~m} \cdot \mathrm{s}^{-1}$ (b)

\section{Sensor em ponte e circuito eletrônico do anemômetro de fio quente \\ O efeito da variação de temperatura sobre o sensor como braço da ponte de Wheatstone é mostrado na Figura 10. Observou-se que, para uma variação de temperatura de $20^{\circ} \mathrm{C}$, o sinal na saída da ponte sofreu variação de $4,0 \mathrm{mV}$, constatando-se a necessidade de compensador de temperatura no circuito do anemômetro.}

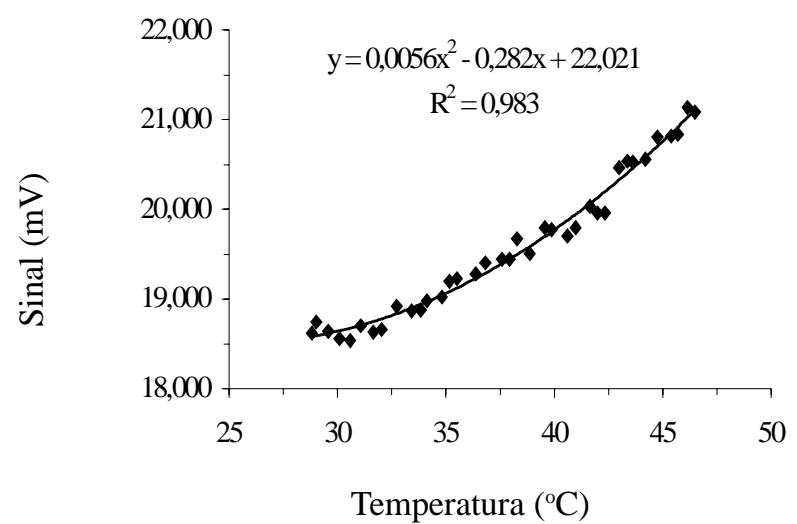

Figura 10. Efeito da variação da temperatura sobre o sensor ligado em ponte de Wheatstone

A tensão na ponte, calculada pela lei de $\mathrm{Ohm}$ em função da corrente no sensor de $160 \mathrm{~mA}$, foi de 5,37V. A resposta do sensor em ponte para um intervalo de velocidade do ar entre 0,00 a $5,00 \mathrm{~m} \cdot \mathrm{s}^{-1}$, alinhada na direção normal ao fluxo, está mostrada na Figura 11.

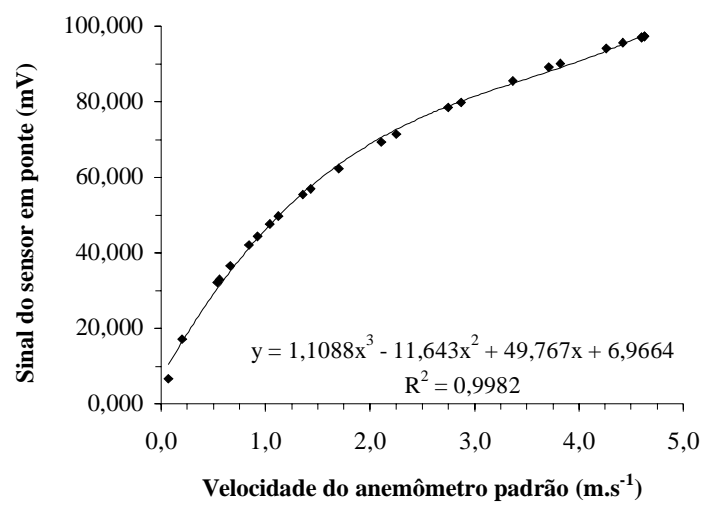

Figura 11. Sinal do sensor em ponte de Wheatstone para velocidades do ar entre 0,0 a $5,00 \mathrm{~m} \cdot \mathrm{s}^{-1}$

Na Figura 11 observa-se que o sensor mostrou alta sensibilidade para velocidades entre 0,00 a $3,40 \mathrm{~m} . \mathrm{s}^{-1} \mathrm{e}$, para velocidades superiores, a sensibilidade diminuiu, sendo que nessas velocidades o alto valor do coeficiente de convecção provoca a saturação do sensor. A equação de regressão que melhor se ajustou aos dados tem coeficiente de determinação ajustado, $R^{2}$, de 0,99 .

Na Figura 12 é mostrada a curva de calibração do protótipo para velocidades entre $0,00 \mathrm{a} 5,00 \mathrm{~m} \cdot \mathrm{s}^{-1}$.

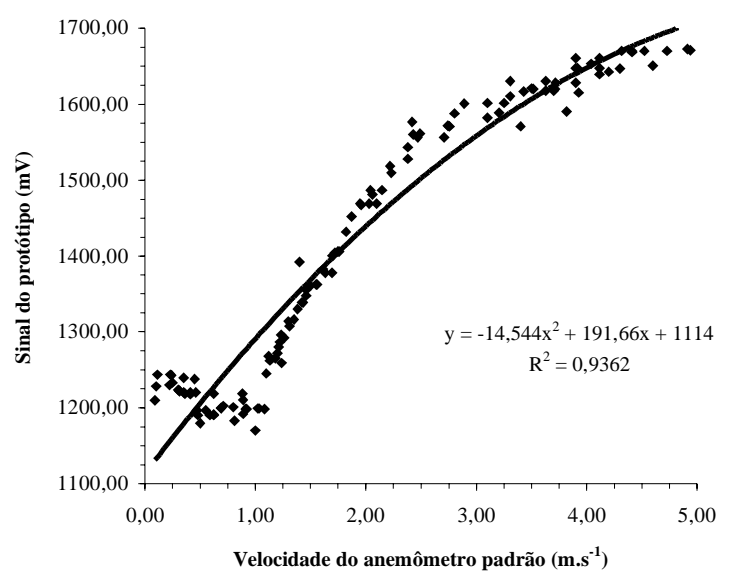

Figura 12. Curva de calibração do protótipo 
O sensor em ponte apresentou variação de tensão de 10,0 $\mathrm{mV}$, enquanto o sinal fornecido pelo operacional é de $500 \mathrm{mV}$, correspondendo a um ganho de 50 vezes. Verificou-se comportamento similar das curvas do sensor em ponte e no circuito eletrônico. Para velocidades inferiores a $1,00 \mathrm{~m} \cdot \mathrm{s}^{-1}$, a sensibilidade do sensor em ponte é superior à do circuito eletrônico. Verificou-se, ainda, falta de estabilidade nas medições, principalmente a baixas velocidades, provenientes, do sensor de tungstênio e do operacional. A não utilização de todas as portas e pinagem do operacional proporcionou o aparecimento de ruídos e de sinais expúrios no circuito.

\section{CONCLUSÕES}

Deste trabalho, podem ser tiradas as seguintes conclusões:

1. Testes com diferentes correntes de aquecimento do filamento levaram à corrente de $160 \mathrm{~mA}$, tendo em vista o compromisso entre vida útil do sensor e sensibilidade para velocidades do ar inferiores a $5,00 \mathrm{~m} \cdot \mathrm{s}^{-1}$.

2. O efeito da variação da temperatura do ar sobre o sensor mostrou a necessidade de compensação de temperatura.

3. Resposta ao cosseno para diferentes velocidades do ar mostrou tolerância quanto à perpendicularidade entre o sensor e o fluxo de ar.

4. O sensor ligado em ponte de Wheatstone teve desempenho considerado satisfatório para medida da velosidade do ar inferiores a $5,00 \mathrm{~m} \cdot \mathrm{s}^{-1}$.

5. O circuito eletrônico de restauração de equilíbrio da ponte de Wheatstone apresentou saturação para velocidade de ar inferior a $1,00 \mathrm{~m} \cdot \mathrm{s}^{-1}$.

6. O sensor ligado com o circuito eletrônico apresentou limitações; assim, faz-se necessário testar outros metais para serem utilizados como sensor.

7. O circuito eletrônico apresentou limitações, sendo necessários ajustes posteriores e emprego de outros tipos de amplificadores operacionais, principalmente para baixas velocidades do ar.

\section{REFERÊNCIAS BIBLIOGRÁFICAS}

CHAMPAGNE, F.H.; SLEICHER, C.A.; WEHRMANN, O.H. Turbulence measurements with inclined hot-wires. Part 1. Heat transfer experiments with inclined hot-wire. Journal Fluid Mechanics, v.28: p.153-175, 1967.

DAVIS, M.R. The dynamic response of constant resistance anemometer. Journal of Physices E.: Scientific Instruments, v.3: p.17-20, 1970.

LOMAS, C.G. Fundamentals of hot wire anemometry. Cambridge, The University of Cambridge, 1986. 211p.

MILLER, I.S.; SHAH, D.A.; ANTONIA, R.A. A constant temperature hot-wire anemometer. Journal of Physices E.: Scientific Instruments, v.20: p.311-314, 1987.

NATIONAL ASSOCIATION OF FAN MANUFACTURES NAFM. Standards, definitions, terms and test codes for centrifugal, axial and propeller fans. 2.ed. Detroit, 1952. (Bulletin, 110).

OWER, E.; PANKHURST, R.C. The measurement of air flow. 5.ed. New York: Pergamon Press, 1977. 374p.

PEARCY, R.W.; EHLERINGER, J.R.; MOONEY, H.A.; RUNDEL, P.W. Plant physiological ecology; field methods and instrumentation. New York: Chapamn and Hall, 1989. 442p.

PERRY, A.E. \& MORRISON, G.L. A study of the constant temperature hot- wire anemometer. Journal of Fluid Mechanics, 47: 577-599, 1971.

PLATT, R.B.; GRIFFITHS, J.F. Environmental measurement and interpretation. 3.ed. New York: Robert E. Krieger, 1972. 231p.

ROSENBERG, N.J.; BLAD, B.L.; VERMA, S.B. Microclimate; the biological environment. 2.ed. New York: John Wiley \& Sons, 1983. 495p.

WATMUFF, J.H. Na investigation of the constant temperature hot-wire anemometer. Experimental Thermal and Fluid Science: 1995.

WEIDMAN, P.D.; BROWAND, F.K. Analysis of a simple circuit for constant temperature anemometry. Journal of Physices E.: Scientific Instruments, v.8: p.533-560, 1975. 